\title{
Kinetics of Host Cell Recruitment During Dissemination of Diffuse Malignant Peritoneal Mesothelioma
}

\author{
Nathan R. Miselis • Bonnie W. Lau • Zhijin Wu • \\ Agnes B. Kane
}

Received: 17 March 2010 / Accepted: 7 June 2010 /Published online: 22 June 2010

(C) The Author(s) 2010. This article is published with open access at Springerlink.com

\begin{abstract}
Diffuse malignant mesothelioma is an aggressive tumor which displays a median survival of 11.2 months and a 5 -year survival of less than $5 \%$ emphasizing the need for more effective treatments. This study uses an orthotopic model of malignant mesothelioma established in syngeneic, immunocompetent $\mathrm{C} 57 \mathrm{Bl} / 6$ mice which produce malignant ascites and solid tumors that accurately replicate the histopathology of the human disease. Host stromal and immune cell accumulation within malignant ascites and solid tumors was determined using immunofluorescent labeling with confocal microscopy and fluorescenceactivated cell sorting. An expression profile of cytokines
\end{abstract}

Electronic supplementary material The online version of this article (doi:10.1007/s12307-010-0048-1) contains supplementary material, which is available to authorized users.

N. R. Miselis $\cdot$ B. W. Lau • A. B. Kane

Department of Pathology and Laboratory Medicine,

Brown University,

Providence, RI, USA

B. W. Lau

e-mail: Bonnie_Lau@BROWN.EDU

A. B. Kane

e-mail: Agnes_Kane@brown.edu

B. W. Lau

Pathobiology Graduate Program, Brown University,

Providence, RI, USA

Z. Wu

Center for Statistical Sciences, Brown University,

Providence, RI, USA

e-mail: ZWU@STAT.BROWN.EDU

N. R. Miselis $(\bowtie)$

Massachusetts General Hospital Cancer Center,

Boston, MA 02115, USA

e-mail: Miselis.Nathan@mgh.harvard.edu and chemokines was produced using quantitative real-time PCR arrays. Tumor spheroids and solid tumors show progressive growth and infiltration with host stromal and immune cells including macrophages, endothelial cells, $\mathrm{CD}^{+}$ and $\mathrm{CD} 8^{+}$lymphocytes, and a novel cell type, myeloid derived suppressor cells (MDSCs). The kinetics of host cell accumulation and inflammatory mediator expression within the tumor ascites divides tumor progression into two distinct phases. The first phase is characterized by progressive macrophage and $\mathrm{T}$ lymphocyte recruitment, with a cytokine profile consistent with regulatory $\mathrm{T}$ lymphocytes differentiation and suppression of $\mathrm{T}$ cell function. The second phase is characterized by decreased expression of macrophage chemotactic and T-cell regulating factors, an increase in MDSCs, and increased expression of several cytokines which stimulate differentiation of MDSCs. This cellular and expression profile suggests a mechanism by which host immune cells promote diffuse malignant mesothelioma progression.

Keywords Malignant mesothelioma - Orthotopic model . Murine $\cdot$ Expression profile $\cdot$ Tumor microenvironment

$\begin{array}{ll}\text { Abbreviations } \\ \text { WT } & \text { C57Bl/6 } \\ \text { eGFP } & \text { C57Bl/6 TgN (bACT-eGFP) 10sb } \\ \text { FGF } & \text { Fibroblast growth factor } \\ \text { FACS } & \text { Fluorescence-activated cell sorting } \\ \text { FGF2 } & \text { Fibroblast growth factor } 2 \\ \text { H\&E } & \text { Hematoxylin and eosin } \\ \text { IL } & \text { Interleukin } \\ \text { i.p. } & \text { Intraperitoneal } \\ \text { MDSCs } & \text { Myeloid-derived suppressor cells } \\ \text { PBS } & \text { Phosphate buffered saline } \\ \text { TGF- } \beta & \text { Transforming growth factor- } \beta \\ \text { VEGF } & \text { Vascular endothelial growth factor }\end{array}$




\section{Background}

Diffuse malignant mesothelioma is an aggressive tumor which is diagnosed at an advanced stage 20 to 60 years following chronic asbestos exposure [1-3]. Diffuse malignant mesothelioma most frequently arises in the pleural, peritoneal or pericardial linings. Malignant ascites is accompanied by solid tumor masses with extensive spreading over the serosal surfaces and local invasion into the lung parenchyma [4]. The prognosis for patients with diffuse malignant mesothelioma is poor with a median survival of 11.2 months and a 5-year survival of less than $5 \%$ [5]. Poor survival is due to the inability to achieve complete surgical resection or eradication by radiotherapy resulting in local tumor recurrence with pleural and peritoneal seeding. Recent clinical studies using multimodality therapies combining radical surgery, multiple agent chemotherapy, and radiotherapy to treat selected patients have increased survival up to a median of 18.1 months with a 5-year survival of about $12 \%[6]$.

The failure of conventional surgical, chemotherapeutic, or radiotherapy protocols directed towards the neoplastic cells $[7,8]$ has motivated the exploration of alternative therapeutic targets. Anti-angiogenic therapies, such as vascular endothelial growth factor (VEGF) inhibitors, have shown some efficacy in diffuse malignant mesothelioma [9], but the most promising adjuvant therapy is immunotherapy. The combination of an activating anti-CD40 antibody [10, 11] or interleukin 2 (IL2) [12] with tumor debulking surgery produces significant improvements in survival with some rodent studies showing a resistance to tumor re-challenge. More complete characterization of the tumor microenvironment is necessary to identify additional host targets for novel and less toxic therapeutic approaches.

The presence of host stromal and immune cells has been linked to aggressive tumor growth, invasion, and metastasis in several types of cancer [13]. The best documented correlation is between density of tumor-associated macrophages and poor prognosis in $80 \%$ of epithelial cancers, as well as in diffuse malignant mesothelioma [14, 15]. Tlymphocytes, macrophages, NK cells, fibroblasts, and dendritic cells have each been identified in human and murine diffuse malignant mesotheliomas [16-18] with studies showing that targeting T-lymphocytes [17, 19] or macrophages [14] delays tumor progression in murine diffuse malignant mesothelioma.

Recruitment, regulation, and activation of host stromal and immune cells is mediated by a complex network of extracellular signals [20]. In the tumor microenvironment, cytokines, angiogenic factors, and growth factors are produced by both tumor and host cells [21, 22]. Human pleural effusions and solid malignant mesotheliomas show elevated expression of VEGF [17, 23], fibroblast growth factor 2 (FGF2)[24], hepatocyte growth factor [25] and IL$6[17,26]$. These factors stimulate mesothelioma cell growth in vitro as well as angiogenesis in vivo [23, 27]. Immunoregulatory factors including transforming growth factor- $\beta$ (TGF- $\beta$ )[28] and IL-10[29] have been shown to reduce T-cell mediated killing of malignant mesothelioma cells [30]. Diffuse malignant mesotheliomas show elevated levels of several chemotactic factors, including CCL5, which recruits macrophages, and CXCL12, which recruits a variety of stromal and immune cells [17]. Alteration to the tumor microenvironment by targeting key immunoregulatory factors and host immune cells has shown the potential to deactivate tumor immunosuppression. Treatment of ectopic murine malignant mesotheliomas with a TGF- $\beta$ receptor kinase inhibitor and interferon- $\beta$ produces a reduction in tumor growth, increases survival and alters host tumor stromal and immune cells [31].

Despite the well-established causal relationship between asbestos and diffuse malignant mesothelioma, cases of early stage disease are extremely rare due to the long and unpredictable latent period and the low incidence of chronically exposed individuals developing the disease [32]. Therefore, studies of early stage diffuse malignant mesothelioma require use of animal models. Autochthonous models of diffuse malignant mesothelioma accurately replicate the natural history and pathology of the human disease, but are unsuitable for most studies due to the long latent period between exposure to asbestos fibers and tumor development [33]. In this study, an orthotopic, immunocompetent murine model is used to characterize the peritoneal microenvironment throughout the course of tumor growth and progression. This characterization of the cellular and expressed microenvironment during tumor progression has identified mediators which have the potential to be critical targets for the development of novel immunotherapies.

\section{Methods}

Cell Culture The malignant mesothelioma cell line, $40 \mathrm{~L}$, was derived from lung micrometastases developed in a C57B1/6 mouse following subcutaneous injection of the primary, asbestos-induced mesothelioma cell line, 40, reported in Goodglick et al. [34]. A non-tumorigenic, immortalized, mesothelial cell line, D9, was derived from a diaphragmatic explant following a single exposure to asbestos as described in the same publication. The cell lines were maintained in DMEM with $2 \mathrm{mM}$ L-glutamine, $1 \mathrm{mM}$ sodium pyruvate, 10 units $/ \mathrm{ml}$ penicillin and $10 \mu \mathrm{g} / \mathrm{ml}$ streptomycin, $10 \mu \mathrm{g} / \mathrm{ml}$ gentamicin, $10 \%$ fetal bovine serum in humidified atmosphere of $94 \%$ air $/ 6 \% \mathrm{CO}_{2}$ at $37^{\circ} \mathrm{C}$. 
Mice Male C57Bl/6 (Wild-type; WT) and C57Bl/6 TgN (bACT-eGFP) 10sb (eGFP) mice were purchased from Jackson Laboratories. All eGFP mice were the offspring of an in-house breeding colony. Mice were housed in sterile rodent HEPA ventilated microisolators (Thoren Units) in a facility approved by the American Association for Accreditation of Laboratory Animal Care that met all federal and state animal care principles, guidelines and regulations. The mice used were between 2 and 12 months of age.

Intraperitoneal Transplantation of Mesothelioma Cells Intraperitoneal (i.p.) injection was used to deliver malignant mesothelioma or immortalized mesothelial cells $\left(2 \times 10^{6}\right)$. Mice were euthanized by $\mathrm{CO}_{2}$ asphyxiation and the organs within the thorax and abdomen were harvested. Prior to organ harvest, the peritoneum of eGFP mice, and some WT mice, was perfused with $4 \%$ paraformaldehyde for $1 \mathrm{~h}$. Tissues were stored at $-80^{\circ} \mathrm{C}$ in OCT blocks and sectioned at $7-10 \mu \mathrm{m}$ using a cryostat. Tissues from WT mice not fixed in paraformaldehyde were removed at necropsy and either fixed in 10\% formalin for assessment of tissue morphology or lysed for RNA and qRT-PCR expression microarray. Tissues fixed in $10 \%$ formalin were paraffin embedded, sectioned at $5 \mu \mathrm{m}$, and stained with hematoxylin and eosin (H\&E).

Tumor spheroids were isolated by lavage from both paraformaldehyde perfused and unfixed mouse peritoneum. Ascitic cells and tumor spheroids were collected by lowspeed centrifugation and flash-frozen in liquid nitrogen for RNA isolation, plated onto glass coverslips, or frozen in OCT as cell blocks. Total RNA was isolated from tissue and lavage cells with TRI-reagent ${ }^{\circledR}$ (Molecular Research Center, Inc., Cincinnati, OH) or with RNeasy mini kit (QIAGEN Inc., Valencia, CA) according to the manufacturer's protocol.

Reagents for Histology Tissues and tumor ascites were harvested on days $7,14,21$, and 28 following injection. Tissues fixed in $10 \%$ formalin were paraffin embedded, sectioned at $5 \mu \mathrm{m}$, and stained with H\&E. Data was compiled according to the day following cell injection and are presented as the mean $+/-$ SEM ( $\mathrm{N} \geq 4$ mice per group). The number and size of tumors was assessed within randomly selected, representative tissue sections using an Axioplan microscope. Tumor ascites harvested following injection with mesothelioma or mesothelial cells were attached for $24 \mathrm{~h}$ on glass coverslips and stained with May-Grünwald and Geimsa stains; frozen into OCT as a $1 \%$ agarose cell block, cryosectioned and immunofluorescent labeled; or lysed for RNA isolation. Micrographs of 5 randomly selected, fields on the May-Grünwald and Geimsa stained slides were examined for each animal to assess spheroid size. The data reported are the longest diameter of 10 randomly selected spheroids from each of three mice per time point. Brightfield micrographs of stained cells were obtained using a Nikon Eclipse E800 microscope with a Spot RT color camera and software.

Reagents for Immunofluorescence Cryosectioned solid tumors and tumor ascites containing spheroids were immunofluorescently labeled for stromal and immune cell markers. Immunolabeling used a primary antibody solution containing one or two of the following: rat-anti mouse F4/ 80 antigen, rat anti-mouse CD68, ALEXA 488-conjugated hamster anti-CD11c, Rat anti-mouse CD4, Rat anti-mouse CD8 $\alpha$, Alexa 488-conjugated Rat anti-mouse CD25, Alexa 488-conjugated Rat anti-mouse CD11b (ABD Serotec, Raleigh, NC), Rat anti-mouse GR-1, Rat anti-mouse CD117 (BD Pharmingen, San Diego, CA), ALEXA 488conjugated rat anti-CD144 (BD Pharmingen, San Diego, CA), rabbit anti-eGFP (Chemicon International, Inc., Temecula, CA) in a blocking solution composed of $10 \%$ normal sera matched to the species of the secondary antibody, $0.1 \%$ Tween- 20 and $0.1 \%$ bovine serum albumin in phosphate buffered saline (PBS). After a $24 \mathrm{~h}$ incubation at $4^{\circ} \mathrm{C}$ the tissue or cells were thoroughly rinsed and incubated in a $5 \mu \mathrm{g} / \mathrm{ml}$ dilution of one or two of the following: ALEXA 555-conjugated goat anti-rat, ALEXA 488-conjugated donkey anti-rabbit IgG or ALEXA 488conjugated donkey anti rat IgG (Invitrogen, Carlsbad, CA) in blocking solution. Omission of the primary antibody was performed in parallel for each sample and confirmed that the observed fluorescence is specific. The tissues or cells were then rinsed with PBS and mounted in Vectashield ${ }^{\circledR}$ hardset containing DAPI (Vector Laboratories, Burlingame, CA). Apoptosis was assessed by TUNEL assay (Roche Diagnostics, Indianapolis, IN) or by nuclear condensation and fragmentation assessed by light microscopy. Confocal fluorescent micrographs were created using a Zeiss 410 confocal laser scanning microscope (Carl Zeiss, Inc., Thornwood, NY) with Phoenix version 2.0 software (Microcosm, Inc., Columbia MD).

Profile of Tumor Ascites Cells During Mesothelioma CellDerived Tumor Formation Tumor spheroids were separated from tumor ascites using low speed (500 RPM for $5 \mathrm{~min}$ ) centrifugation and dissociated into single cell suspension with collagenase/trypsin and agitation. The "free cell" and "spheroids-associated" cell fractions were paraformaldehyde fixed and immunofluorescently labeled to identify stromal and immune cells as described above. Paired unstained samples were used as negative controls. Fluorescence-activated cell sorting (FACS) was performed to profile the ascitic cells over the time course of tumor spheroid formation. A FACSCalibur ${ }^{\mathrm{TM}}$ system (Becton Dickinson, San Jose, CA) was used for sorting and CELL 
Quest software (Becton Dickinson) was used to perform the analysis. Data were compiled temporally and the fraction of the total cells composed by each cell type for each mouse ( $\mathrm{N} \geq 2$ mice per group) is displayed.

$R T^{2}$ Profiler $^{\mathrm{TM}}$ PCR Arrays Total RNA $(1 \mu \mathrm{g})$ was harvested from peritoneal exudate cells before (resident peritoneal cells), 7, 14 and 21 days following i.p. injection of mesothelioma cells, from solid tumors harvested 21 days following i.p. injection, as well as from mesothelioma and mesothelial cells grown in vitro. A quantitative real-time PCR array was used to screen inflammatory cytokines and receptors (Cat. No. PAMM-011), angiogenic factors (Cat. No. PAMM-024) and tumor metastasis-related transcripts (Cat. No. PAMM-028) according to the manufacturer's instructions (SuperArray Bioscience, Frederick, MD). Reactions were cycled in an ABI Prism ${ }^{\circledR} 7500$ FAST sequence detector (Applied Biosystems, Inc., Foster City, $\mathrm{CA}$ ) and the acquired data was analyzed to determine the relative expression level of each transcript after normalization to the expression level of housekeeping gene controls.

Statistics All data, excepting the FACs data, were calculated as the mean \pm SE. The number and area of tumors were determined using representative tissue sections from each of seven sites within the peritoneal cavity. The sum of all tumor areas within each mouse is expressed as the total tumor burden. Statistical significance was assessed using the following methods with differences with $P<0.05$ being considered statistically significant. The significance of variations within the increasing trend to spheroid size, tumor frequency and tumor burden was assessed by linear regression or Poisson regression (Figs. 1 and 2). Significant changes in expression over time (Fig. 5) were determined using a moderated $t$-test [35] with significance level 0.01(Benjamini-Hochberg false discovery rate).

\section{Results}

Pathology of Transplanted Murine Malignant Mesothelioma

Orthotopic transplantation of an asbestos-induced mesothelioma cell line into the peritoneum of syngeneic mice reproducibly produces tumor ascites, tumor spheroids, and solid tumors that re-capitulate the human disease (Figs. 1 and 2). As early as 7 days following intraperitoneal injection of mesothelioma cells, tumor spheroids (Fig. 1b) and microscopic tumor masses are established (Fig. 2b, first panel). Multicellular spheroids are present both before and after formation of solid tumors indicating a potential role in both tumor establishment and the spread of tumor on the serosal surfaces. Early solid tumors (Fig. 2b, third panel) are microscopic aggregates of tumor and host stromal cells that attach to the mesothelial lining. Vascularized tumor masses attach on the inferior surface of the diaphragm and
Fig. 1 Kinetics of tumor spheroid growth. a. Average area of tumor spheroids within malignant ascites over time \pm SEM $(N \geq 30)$. b. Representative spheroids shown by cell type and time point (Bar=100 um) with a representative image of apoptotic cells (Bottom, fourth panel; Bar $=50$ um)



b.

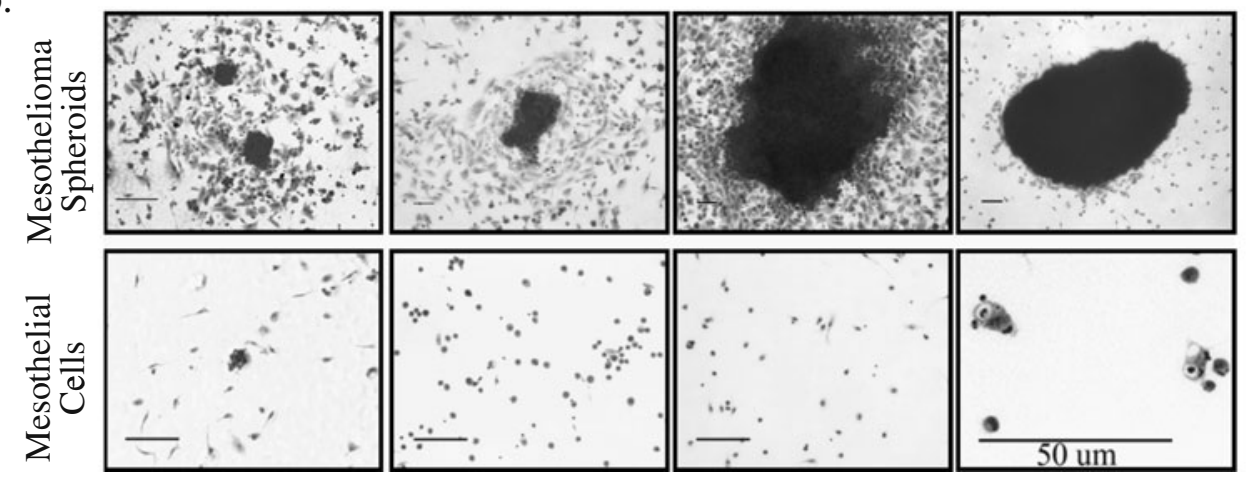


Fig. 2 Kinetics of tumor mass dissemination and growth.

a. Average tumor frequency (top) and tumor burden (bottom) following mesothelioma cell injection. Each mean was calculated from at least three mice and is shown \pm SEM. $\mathbf{b}$. Representative images of tumor growth and progression. Freefloating tumor spheroids (second panel) are composed of malignant (circles) and host stromal cells (arrowheads). Tumor masses show a progressive pattern of tumor establishment (first panel), growth (third and fourth panels) and invasion into adjacent tissues (fourth panel). H\&E stain


b.

Day 14

Day 14

Day 21

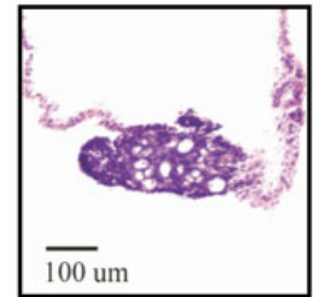

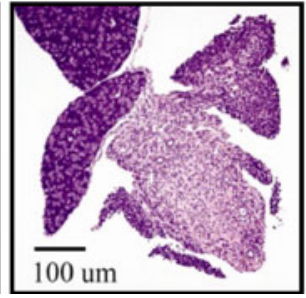

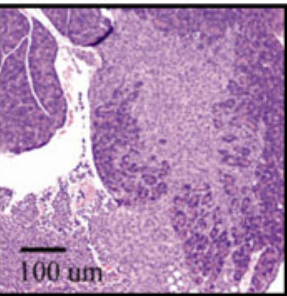

on the mesentery. After 21 days, solid tumor masses display invasive growth penetrating into the parenchyma of abdominal organs (Fig. 2b, fourth panel).

The distinct and reproducible pattern of tumor seeding, implantation, and invasion makes this tumor model well suited for kinetic studies of the tumor microenvironment during tumor progression. Injection of malignant mesothelioma cells produces spheroids which grow progressively up to 21 days when the average size nears $0.1 \mathrm{~mm}^{2}$ (Fig. 1a, black bars) and a characteristic necrotic core forms at the center of the tumor spheroids (data not shown). In contrast immortalized, non-tumorigenic mesothelial cells form very small aggregates that disappear after 14 days (Fig. 1a, white bars). The inability of immortalized, nontumorigenic mesothelial cells to form viable cell aggregates corresponds with a higher incidence of apoptosis when compared to injection of malignant mesothelioma cells, 8times greater at day $7(3.2 \%$ vs. 0.4$)$, 2-times greater at day $14(1.4 \%$ vs. $0.7 \%)$, and more than 4-times greater at day $21(3.3 \%$ vs. $0.7 \%)$.

The kinetics of solid tumor establishment and growth is different from that of tumor spheroids. The frequency of solid tumors within the peritoneal cavity gradually increases throughout 28 days (Fig. 2a, top), while the total tumor area (tumor burden) shows a delayed progression (Fig. 2a, bottom). Between 7 and 14 days post-tumor cell injection the tumor burden increases 2-fold from $2.1 \mathrm{~mm}^{2}$ to $5.4 \mathrm{~mm}^{2}$ with a similar 2-fold increase between days 21 and 28; however between day 14 and 21 the tumor burden increases 8-fold from $5.4 \mathrm{~mm}^{2}$ to $43.9 \mathrm{~mm}^{2}$. During this accelerated phase of tumor development, there is increased tumor invasion into adjacent tissues (Fig. 2b, fourth panel) followed by development of pulmonary metastases [14].

Identification of Host Stromal and Immune Cells in Spheroids and Solid Tumors

Similar to the human disease [16-18], this tumor model shows infiltration of host stromal and immune cells into both tumor spheroids and solid tumors. Transplantation of mesothelioma cells into eGFP mice reveals a significant proportion of host cells in both tumor spheroids (Fig. 3a, first panel) and solid tumors (Fig. 3b, first panel). Immunofluorescent labeling of spheroids and tumors established in wild-type mice identifies 
diffusely distributed tumor-associated macrophages (Fig. 3a and $b$, second panels) and endothelial cells which are present in tumor spheroids as isolated cells that form an extensive vascular network in solid tumors (Fig. 3a and b, third panel). Myeloid-derived suppressor cells (MDSCs), identified by
GR-1 (red fluorescence) and CD11b (green fluorescence) expression, are present in spheroids but are less frequent in solid tumors (Fig. 3a and b, fourth panel, arrowheads). Other immune cells, albeit at low density, include dendritic cells (Fig. 3c and d, first panel), $\mathrm{CD} 4^{+}, \mathrm{CD} 25^{+}$lymphocytes
All Stromal cells (eGFP)

a.



b.

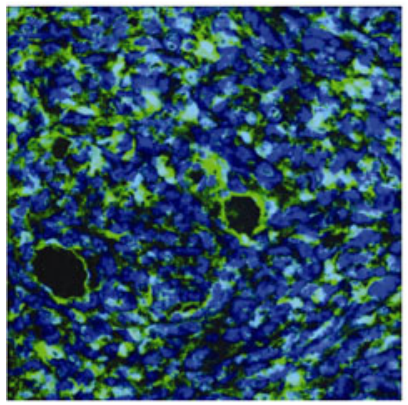

Dendritic Cells

(CD11c)

c.



d.

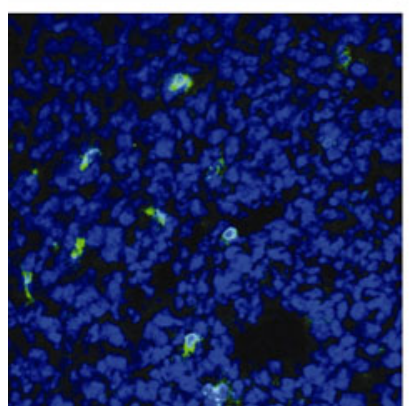

Tumor-Associated Macrophages

(F4/80)
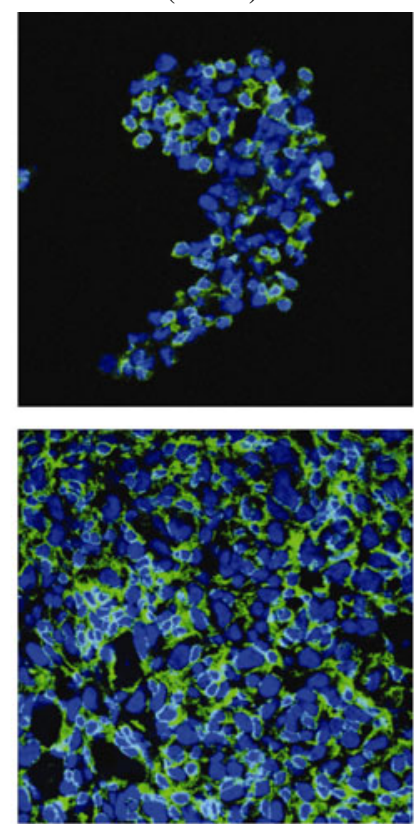

Regulatory T lymphocytes

(CD4 \& CD25)
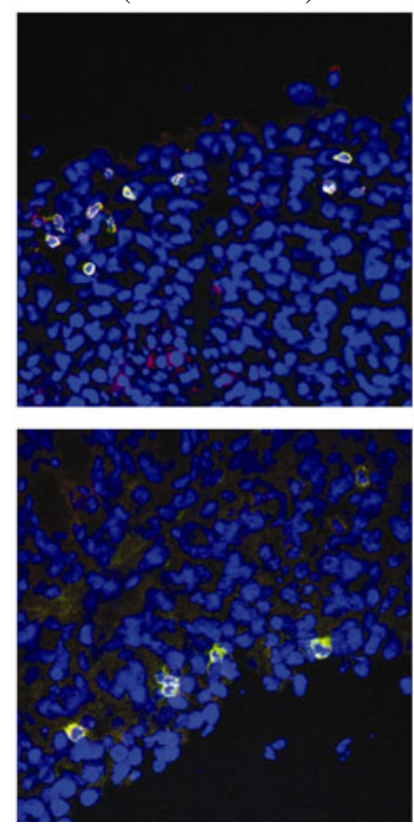

Endothelial cells

(CD144)
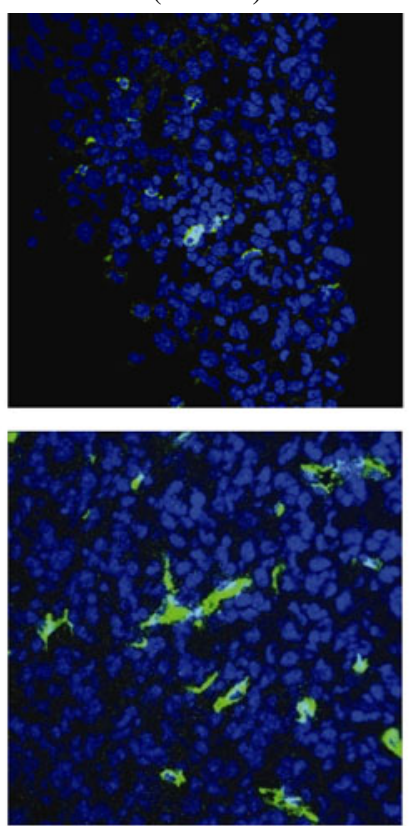

Cytotoxic T lymphocytes (CD3 \& CD8)
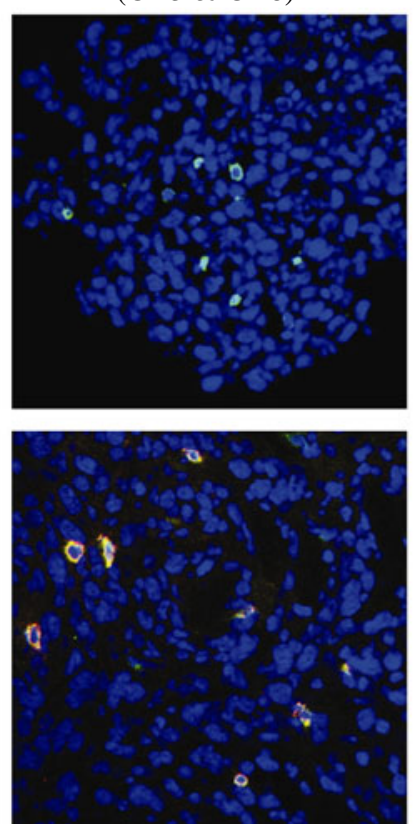

MDSCs

(CD11b \& GR-1)
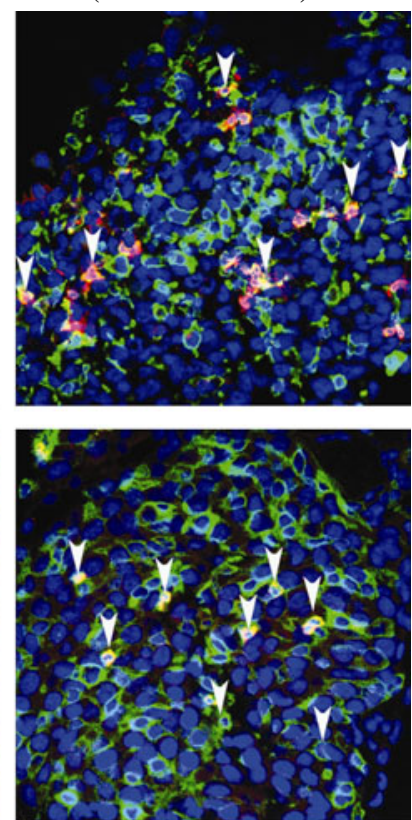

Haemotopoietic stem cells

(CD117)


Fig. 3 Host stromal cells in tumor spheroids and solid tumors. Identification of host cells within tumor spheroids $(\mathbf{a}, \mathbf{c})$ and solid tumors (b, d). Indirect immunofluorescent labeling for eGFP identifies host-derived cells, F4/80 identifies macrophages and CD144 identifies endothelial cells. Expression of CD11b and GR-1 identifies myeloid- derived suppressor cells (white arrowheads), CD11c identifies dendritic cells, CD4 and CD25 identifies regulatory T lymphocytes, and CD3 and CD8 identifies cytotoxic T lymphocytes. Following immunolabeling, tissues were counterstained with DAPI and assessed by confocal microscopy at 600x magnification 
(Fig. 3c and c, second panel) and $\mathrm{CD}^{+}$lymphocytes (Fig. 3c and $\mathrm{d}$, third panel) and haematopoietic stem and/or endothelial progenitor cells (Fig. 3c and d, fourth panel). In each panel, omission of the primary antibody did not produce fluorescent labeling (data not shown).

Host Stromal and Immune Cell Recruitment to the Peritoneal Cavity and Tumor Spheroids

The presence of host stromal cells within peritoneal tumor spheroids suggests that these cells facilitate tumor spheroid formation, tumor cell survival and growth; therefore, the kinetics of host cell recruitment to tumor ascites and tumor spheroids was examined. Free-floating single cells and tumor spheroid-associated cells were separated from peritoneal lavage fluid and examined by FACS analysis (Fig. 4). Macrophages are the predominant host cell type in recovered ascitic fluid, $45.5 \%$, and in spheroids, 27\%, at 21 days post injection (Fig. 4a). Endothelial cells are present early in the ascitic fluid but decline rapidly and increase to a maximum of $15 \%$ in tumor spheroids at 21 days (Fig. 4b). Dendritic cells
Fig. 4 A quantitative profile of the host cell population within peritoneal effusions and tumor spheroids throughout tumor progression. Free-floating single cells and dissociated spheroidassociated cells were harvested at 7 day intervals from 2 replicate mice and analyzed by flow cytometry to determine the proportion of (a) macrophages, (b) endothelial cells and (c) myeloid derived suppressor cells. The graphs show the data from each mouse ( $\square$ ) and a mean of the replicates (-) a

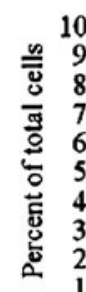

100
90
80
70
60
50
40
30
20
10
0

Macrophage (CD68)

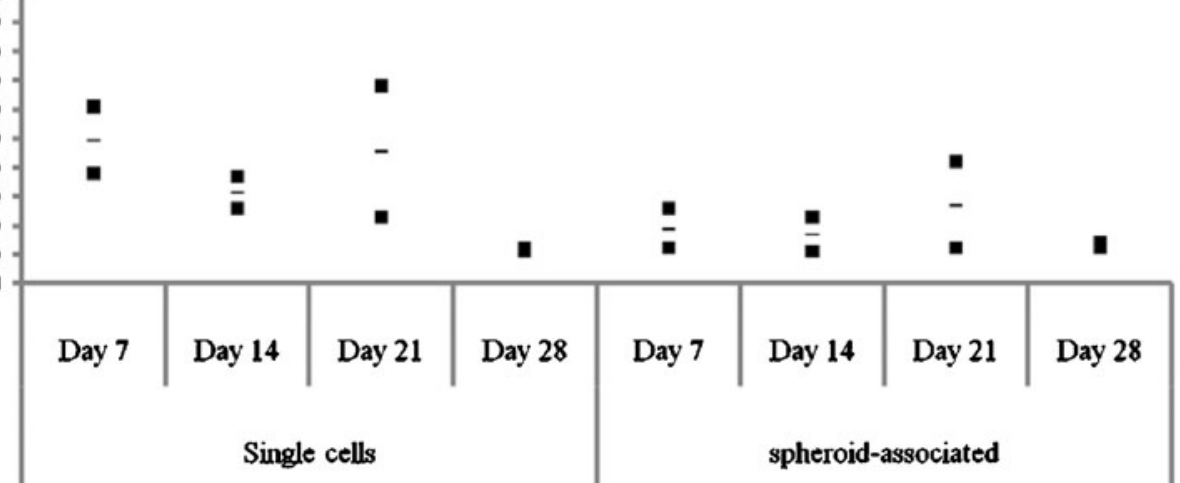

b



Endothelial cells (CD144)



C

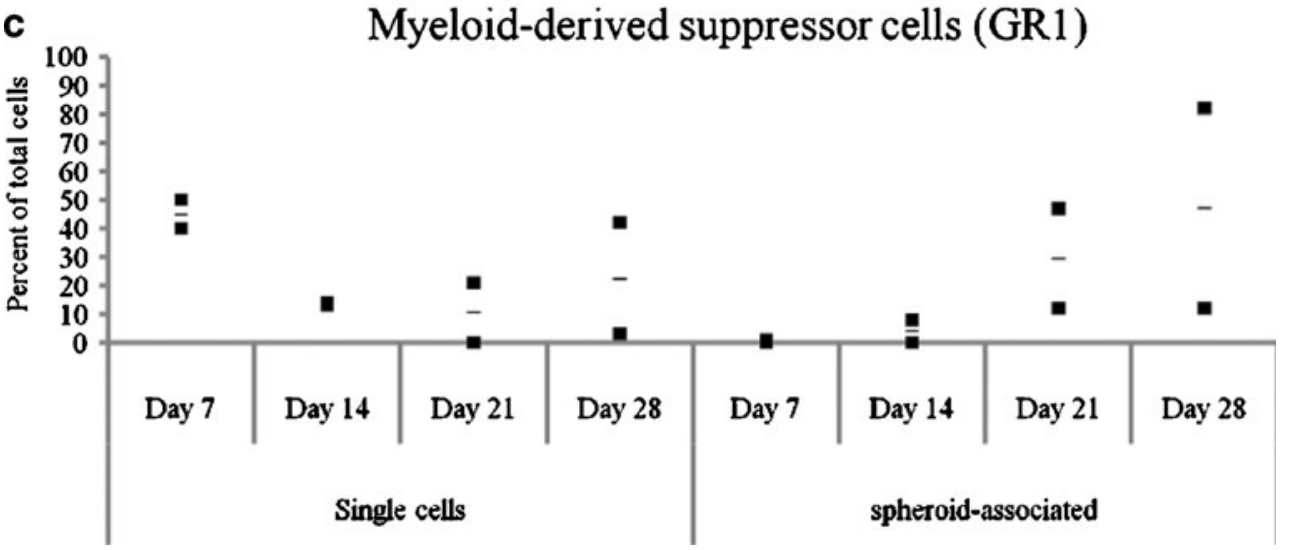


constitute less than $3 \%$ of cells at all time points in both groups (data not shown). In contrast, MDSCs constitute $45 \%$ of the ascitic cells after 7 days, but are not present in tumor spheroids until 14 days (Fig. 4c). The proportion of MDSCs within tumor spheroids shows a progressive increase to a maximum of $47 \%$ after 28 days (Fig. 4c). This trend closely mirrors that of tumor burden (Fig. 2a, bottom) suggesting that spheroid-associated MDSCs could facilitate tumor growth.

Kinetics of Chemokine and Cytokine Expression in Tumor Ascites

To determine the kinetics of host cell recruitment to developing tumors, the gene expression profile of chemokines, cytokines and growth factors was determined throughout tumor establishment and progression (Fig. 5). The expression profile of tumor ascites harvested 7,14 and 21 days post-injection was compared to the malignant mesothelioma cell line in vitro, immmortalized mesothelial cells in vitro, and resident peritoneal cells (Supplemental Table 1). The relative expression is derived from the $\mathrm{Ct}$ values of three biological replicates normalized to the average level of expression for multiple housekeeping genes.
Many chemokines associated with recruitment of host immune cells showed elevated expression as compared to malignant mesothelioma cells in vitro (Fig. 5a and Supplemental Table 1). The most highly expressed chemokines are CCL5, CCL6, and CCR2 which mediate recruitment of monocytes in addition to lymphocytes. Regulatory $\mathrm{T}$ lymphocytes have been suggested to facilitate diffuse malignant mesothelioma progression [17]. While ascitic cells show low expression of CCL20, CCR8, CCL22 and CCR7, there is significantly elevated expression of CCL24 and IL-16 after 7 days with a significant reduction after 14 days(Fig. 5a and Supplemental Table 1).

Many macrophage chemotactic factors are upregulated throughout the course of tumor progression [14]. The most highly expressed chemotactic factors within the tumor ascites are CCL6 and CCL9 with CCL2, CCL5 and CXCL2 showing small increases in expression as compared to the malignant mesothelioma cell line in vitro (Fig. 5a and Supplemental Table 1). The receptors for these cytokines also show greater expression in ascitic cells. CCR1, a receptor for CCL5, CCL6 and CCL9, CCR2, the receptor for CCL2, and CCR5, another receptor for CCL5, all show a significant increase in expression when compared to malignant mesothelioma or immortalized mesothelial cells in vitro (Supplemental Table 1). The chemokines and a
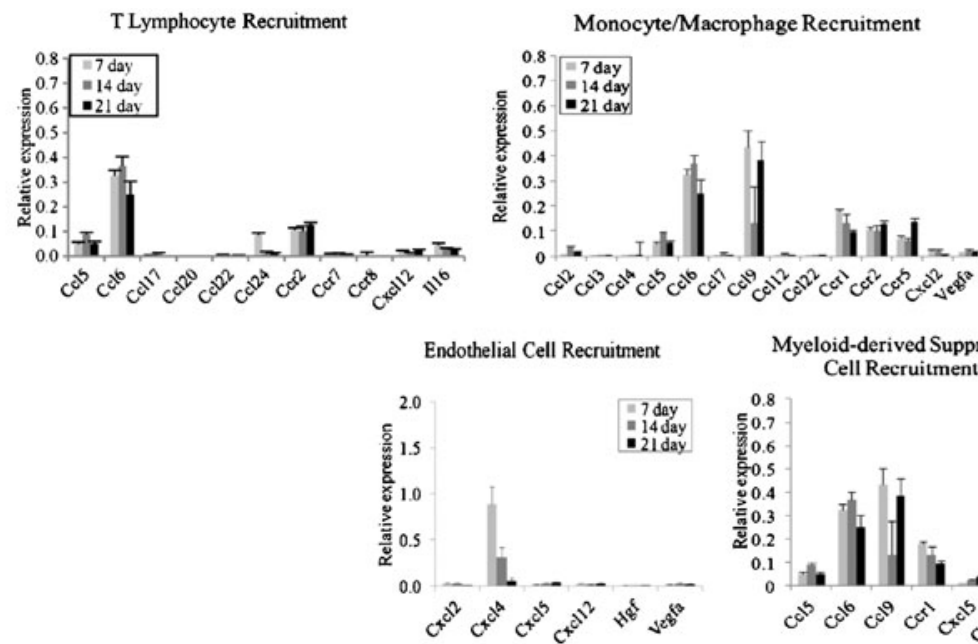



b


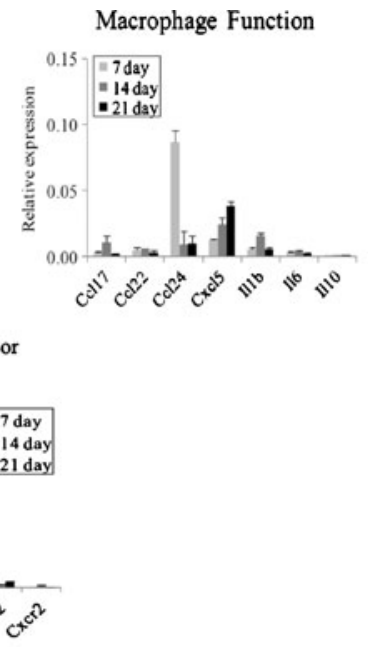

Fig. 5 Profile of angiogenic and inflammatory mediators expressed within peritoneal tumor ascites during tumor progression. Transcripts are grouped by function (a) and time of peak expression (b). The relative level of expression for each group is displayed graphically and is defined as the $2^{\wedge}-\Delta \mathrm{Ct}$ values $+/-\mathrm{SEM} ; N=3$ 
cytokines CCL22, CCL24, CXCL5, IL1b, IL6 and IL10 which are expressed by macrophages show low expression, but is greater than that of malignant mesothelioma or immortalized mesothelial cells in vitro (Fig. 5a and Supplemental Table 1).

Several factors which regulate endothelial cell recruitment are expressed in ascitic cells. The most highly expressed is the anti-angiogenic chemokine CXCL4 which shows a statistically significant trend of decreasing expression throughout tumor progression (Fig. 5a and Supplemental Table 1). The chemokines CXCL2 and CXCL5 show low levels of expression in tumor ascites which are greater than that of the malignant mesothelioma cell line in vitro (Fig. 5a and Supplemental Table 1). Several chemokines for immature myeloid cells and MDSCs are also expressed. As discussed previously, CCL5, CCL6, CCL9, and CXCL5 all showed elevated expression, as did one of the complementary receptors, CCR1.

To assess the kinetics of stromal cells recruitment, transcripts were grouped temporally according to the time of peak expression (Fig. 5b). Transcripts with highest expression after 7 days include CCR1, CXCL4, CXCL13, CXCR5, IL16, TGF $\beta 1$, TGF $\beta 2$ and Thbs1, but also genes that show a rapid decrease, such as CCL24. By day 14 the chemokines that show peak expression are primarily monocyte chemokines and macrophage-derived cytokines (CCL2, CCL5, CCL6, CCL7, CCL17, CCL22, CXCR2, IL1 $\beta$, IL6, SPP1 and VEGFA) and the majority of these chemokines and cytokines show a drop in expression after 21 days(Fig. 5b and Supplemental Table 1). This occurs in conjunction with a decrease in macrophages and increased tumor burden by day 28 (Fig. 4). An inverse trend is observed for the majority of chemokines and cytokines which show their highest level of expression at day 21 . CCL9, CCR2, CCR3, CCR5, Ctgf, CXCL12, Fgf1, MMP2, TGF 33 , Tnfrsfla and Tnfrsflb each show their lowest expression at day 14 and all except CCL9 peak at day 21 (Fig. 5b and Supplemental Table 1). To confirm that these alterations in gene expression corresponded with protein expression and secretion a protein array was performed using peritoneal lavage fluid. Six cytokines, CCL2, CCL5, CCL12, CXCL1, IL-1 $\alpha$ and IL-6, peaked at 14 days in agreement with their gene expression profiles.

Gene Expression Profiles of Murine and Human Diffuse Malignant Mesotheliomas

To evaluate the similarity in the microenvironment within tumor spheroids and solid tumors, the entire expression profiles of cytokines and their receptors were compared. Similar up- or down-regulation was identified for $85 \%$ of the transcripts (71 of 84 ) when shown relative to the mesothelioma cell line in vitro (Fig. 6a). The expression a

\begin{tabular}{|c|c|}
\hline Transcripts showing similar regulation & $\begin{array}{l}\text { Transcripts showing } \\
\text { different regulation }\end{array}$ \\
\hline  & $\begin{array}{l}\text { Bc16, Ccl2, Ccl7, Ccl11, } \\
\text { Ccl19, Il1f6, Il1f8, Il3, } \\
\text { Il4, Il13, Il17b, Il20, } \\
\text { Spp1, }\end{array}$ \\
\hline
\end{tabular}



b

\begin{tabular}{|l|l|}
\hline \multicolumn{1}{|c|}{ Transcripts showing similar regulation } & \multicolumn{1}{|c|}{$\begin{array}{c}\text { Transcripts showing } \\
\text { different regulation }\end{array}$} \\
\hline C3,Ccl1, Cc15, Cc16, Cc111, Ccl17, Ccl19, Ccr1, & Apc, Casp1, Ccl20, Ccl22, \\
Ccr5, Cdh11, Csf1, Ctnna1, Ctsk, Ctsl, Cxcl11, & Ccr7, Cd44, Cdkn2a, Chd4, \\
Cxcr2, Cxcr5, Ela2, Ewsr1, Hras1, Igf1, Il6st, Il10, & Cx3cl1, Cxc14, Cxcl12, Denr, \\
Il10ra, Il10rb, Il13, Il16, Itgam,, Kras, Met, Mmp7, & Fgfr4, Fn1, Il1a, I1112, Il18, \\
Myc, Nme1, Nr4a3,Pnn, Pten, Scye1, Smad2, Syk, & Ltb, Mmp2, Mmp11, Plaur, \\
Tnf, Tnfsf10, Tshr & Tmp3 \\
\hline
\end{tabular}

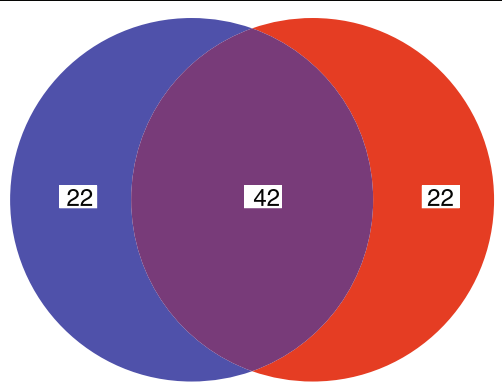

Fig. 6 Alignment of tumor and ascites expression profiles. a. Expression of cytokines, chemokines and their receptors within murine tumors and tumor ascites at 21 days shows similar up- or downregulation relative to the mesothelioma cell line in vitro; $\mathrm{N} \geq 2$. b. Human malignant mesotheliomas and murine malignant mesotheliomas show similar regulation of chemokines, cytokines and metastasis-related transcripts relative to species matched cell lines in vitro; $N \geq 1$

profiles of human diffuse malignant mesotheliomas are highly variable; however, a profile of 64 cytokine, cytokine receptor and metastasis-related transcripts was used to compare the microenvironment of murine malignant mesothelioma after 21 days with human epithelial diffuse malignant pleural mesotheliomas whose expression profile was reported in Mohr, S. et al. [36]. Similar to our comparison of tumor spheroids and solid tumors, these authors compare expression in human malignant mesotheliomas to cell lines in vitro. This analysis shows that $66 \%$ of the transcripts (42 of 64) within murine diffuse malignant mesotheliomas display similar alterations in expression compared to human epithelial diffuse malignant pleural mesotheliomas (Fig. 6b). 


\section{Discussion}

This model of transplanted diffuse malignant mesothelioma shows the formation of malignant ascites, tumor masses and secondary pulmonary metastases similar to autochthonous models of diffuse malignant mesothelioma in rodents and the human disease [19, 34, 37-39] (Figs. 1 and 2). Despite being of murine in origin, the combination of similar disease pathology and gene expression profile (Fig. 6) validates that these orthotopically transplanted diffuse malignant mesotheliomas reflect the microenvironment of the human disease. Using this tumor model, we showed previously that depletion of tumor-associated macrophages significantly reduces tumor growth and metastasis [14]. By profiling host cell recruitment throughout the course of tumor progression, an additional myeloid-derived cell population not previously described in diffuse malignant mesotheliomas was identified: MDSCs. The parallel between increasing tumor burden (Fig. 2a, bottom) and accumulation of MDSCs within tumor spheroids (Fig. 4c) suggests that MDSCs accelerate tumor progression. MDSCs have been reported to mediate the suppression of host acquired immunity and stimulation of tumor growth in other tumor models [40-43] suggesting that MDSCs are participating in the establishment of an immunosuppressive tumor microenvironment in this tumor model.

The presence of MDSCs within tumor spheroids and solid tumors of animals with advanced stage disease identifies this cell type a potential mediator of the nonimmunogenic phenotype of this tumor. MDSCs have been shown to inhibit CD4+ and CD8+ T cell proliferation [42], block natural killer cell activation [43], limit dendritic cell maturation [41] and polarize macrophage towards an M2 phenotype both with and without TGF- $\beta$ expression [4446]. These data suggest strategies that target not only $\mathrm{T}$ lymphocytes, but also macrophages and myeloid-derived suppressor cells could enhance host cell-mediated immune destruction of malignant mesotheliomas.

These results also support a role for regulatory $\mathrm{T}$ cells in suppressing immune responses as proposed by Hegmans et al. 2006 in their study of mouse and human malignant mesotheliomas [17]. Regulatory $\mathrm{T}$ lymphocytes are found within tumor spheroids and solid tumors (Fig. 3c and d, third panels) and appear to be actively recruited and regulated by host chemokines (Figs. 5 and 7a). The majority of lymphocyte chemotactic factors show their highest expression 7 days following injection of tumor cells. CCL24, which recruits resting $\mathrm{T}$ lymphocytes while also suppressing their Th1 immune responses [47], peaks at 7 days. IL-16, which is frequently present in malignant pleural effusions, attracts $\mathrm{T}$ lymphocytes and stimulates CD25 expression during differentiation to regulatory $\mathrm{T}$ lymphocytes [48], also shows a peak at 7 days (Fig. 5b). The cytokine profile of the microenvironment within the peritoneal cavity at this early stage of tumor development is composed of chemokines responsible for recruiting lymphocytes, monocytes, and establishing a local immunosuppressive environment (Figs. 5 and 7a). This evidence suggests that following injection regulatory $\mathrm{T}$ lymphocytes establish an environment of immunotolerance allowing persistence of malignant mesothelioma cells.

This cytokine profile also reveals a prominent role for macrophages. The profile aligns with that of a myeloidenriched tumor "stromal" profile as described by Lenz, et al.[49]. Increased expression of Ctgf, Itgb2, TGF $\beta 1$, Thrombospondin 1 and TIMP2 and decreased expression of Kdr, PECAM1, or TEK is consistent with a myeloidenriched profile rather than an endothelial cell-enriched profile (Fig. 5 and Supplemental Table 1). This characterization is supported by the cellular profile which shows a
Fig. 7 Regulation of the tumor microenvironment. Expressed mediators which participate in the recruitment and regulation of stromal and immune cells at a. 14 days b. 21 days post tumor cell injection
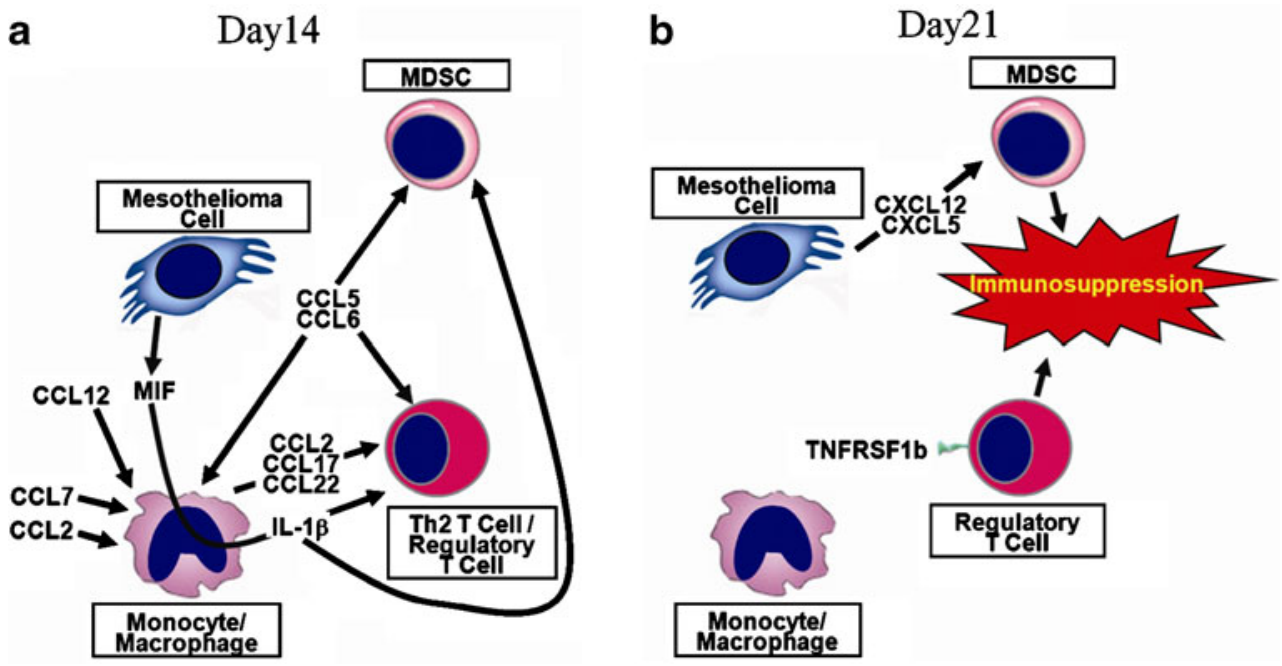
prominent macrophage population throughout tumor progression (Fig. 4a) and the expression profile of monocyte chemotactic factors CCL9, showing peak expression at 7 days, and CCL2, CCL7 and CCL12, that show both statistically significant patterns of expression and peak expression at day 14 (Fig. 5b). A role for macrophages is suggested by the expression of factors which mediate macrophage-lymphocyte interactions (Fig. 5b). For example, the macrophage-derived chemokines, CCL17 and CCL22, which show peak expression at day 14, recruit Th2 and regulatory T-cells and in combination with CCL2 stimulate naïve and resting $\mathrm{T}$ lymphocytes to become regulatory $\mathrm{T}$ lymphocytes $[20,50,51]$ suggesting that macrophages mediate the recruitment and regulation of $\mathrm{T}$ lymphocytes (Fig. 7a).

The cytokine profile three weeks after injection characterizes a microenvironment that suppresses immune surveillance and antitumor immunity (Fig. 7b). CXCL12, a chemotactic factor for MDSC [17], peaks after three weeks. In combination with elevated expression of CCL9 [52] and CXCL5[46] this profile could mediate the substantial increase in MDSCs observed after 3 to 4 weeks (Figs. 4 and $5 b)$.

This publication is the first to present a comprehensive expression profile of the tumor microenvironment throughout the progression of diffuse malignant mesothelioma in a murine orthotopic model. This model was validated by comparing the expression profile of human diffuse malignant mesotheliomas and transplanted murine malignant mesotheliomas which revealed $66 \%$ of the transcripts display similar patterns of expression when compared to species-matched mesothelioma cell lines in vitro (Fig. 6b). The common transcripts describe a microenvironment that actively recruits MDSCs, macrophages and $\mathrm{T}$ lymphocytes. The macrophage and MDSCchemotactic factors, CCL1, CCL5, CCL6, and their receptors CCR1 and CCR5 showed similar regulation in both the human and murine malignant mesotheliomas in addition to $\mathrm{T}$ lymphocyte chemotactic factors CCL1, CCL5, CCL6, and CCL17. These results validate that this murine model of diffuse malignant mesothelioma replicates the human host stromal and immune microenvironment potentially identifying critical cellular targets for novel adjuvant therapies.

Acknowledgements We thank N. Messier for her expertise and assistance in real time PCR. National Institutes of Health for grants RO1 ES03721, T32 ES07272 and F30 ES013639. We acknowledge the contributions of the Molecular Pathology Core Facility established with support from Superfund Basic Research Grant P42 ES013660 which provided assistance with preparation of histopathological samples.

Competing interests The authors have no competing interests, financial or otherwise, to declare.
Open Access This article is distributed under the terms of the Creative Commons Attribution Noncommercial License which permits any noncommercial use, distribution, and reproduction in any medium, provided the original author(s) and source are credited.

\section{References}

1. Mallory TB, Castleman B, Parris EE (1947) Case reports of the Massachussetts general hospital \#33111. N Engl J Med 236:407-412

2. Weldler HW (1943) Uber den Lugenhrebs bei asbestose. Dtsch Arch Klin Med 191:189-209

3. Marinaccio A, Branchi C, Massari S et al (2006) National epidemiologic surveillance systems of asbestos-related disease and the exposed workers register. Med Lav 97:482-7

4. Roggli VL, Oury TD, Sporn TA (2004) Pathology of asbestosassociated diseases. Springer, New York

5. Nowak AK, Byrne MJ, Williamson R et al (2002) A multicentre phase II study of cisplatin and gemcitabine for malignant mesothelioma. Br J Cancer 87:491-6

6. Lee TT, Everett DL, Shu HK et al (2002) Radical pleurectomy/ decortication and intraoperative radiotherapy followed by conformal radiation with or without chemotherapy for malignant pleural mesothelioma. J Thorac Cardiovasc Surg 124:1183-9

7. Rusch VW (1997) Pleurectomy/decortication in the setting of multimodality treatment for diffuse malignant pleural mesothelioma. Semin Thorac Cardiovasc Surg 9:367-72

8. Pass HI, Kranda K, Temeck BK et al (1997) Surgically debulked malignant pleural mesothelioma: results and prognostic factors. Ann Surg Oncol 4:215-22

9. Li Q, Yano S, Ogino H et al (2007) The therapeutic efficacy of anti vascular endothelial growth factor antibody, bevacizumab, and pemetrexed against orthotopically implanted human pleural mesothelioma cells in severe combined immunodeficient mice. Clin Cancer Res 13:5918-25

10. Broomfield S, Currie A, van der Most RG et al (2005) Partial, but not complete, tumor-debulking surgery promotes protective antitumor memory when combined with chemotherapy and adjuvant immunotherapy. Cancer Res 65:7580-4

11. Nowak AK, Robinson BW, Lake RA (2003) Synergy between chemotherapy and immunotherapy in the treatment of established murine solid tumors. Cancer Res 63:4490-6

12. Lucchi M, Chella A, Melfi F et al (2007) A phase II study of intrapleural immuno-chemotherapy, pleurectomy/decortication, radiotherapy, systemic chemotherapy and long-term subcutaneous IL-2 in stage II-III malignant pleural mesothelioma. Eur J Cardiothorac Surg 31:529-33, discussion 533-4

13. Li H, Fan X, Houghton J (2007) Tumor microenvironment: the role of the tumor stroma in cancer. J Cell Biochem 101:805-15

14. Miselis NR, Wu ZJ, Van Rooijen N et al (2008) Targeting tumorassociated macrophages in an orthotopic murine model of diffuse malignant mesothelioma. Mol Cancer Ther 7:788-99

15. Bingle L, Brown NJ, Lewis CE (2002) The role of tumourassociated macrophages in tumour progression: implications for new anticancer therapies. J Pathol 196:254-65

16. Martarelli D, Catalano A, Procopio A et al (2006) Characterization of human malignant mesothelioma cell lines orthotopically implanted in the pleural cavity of immunodeficient mice for their ability to grow and form metastasis. BMC Cancer 6:130

17. Hegmans JP, Hemmes A, Hammad H et al (2006) Mesothelioma environment comprises cytokines and T-regulatory cells that suppress immune responses. Eur Respir J 27:1086-95

18. Davidson B, Dong HP, Holth A et al (2007) Chemokine receptors are infrequently expressed in malignant and benign mesothelial cells. Am J Clin Pathol 127:752-9 
19. Cordier Kellerman L, Valeyrie L, Fernandez N et al (2003) Regression of AK7 malignant mesothelioma established in immunocompetent mice following intratumoral gene transfer of interferon gamma. Cancer Gene Ther 10:481-90

20. Vicari AP, Caux C (2002) Chemokines in cancer. Cytokine Growth Factor Rev 13:143-54

21. Dvorak HF (2005) Angiogenesis: update 2005. J Thromb Haemost 3:1835-42

22. Albini A, Sporn MB (2007) The tumour microenvironment as a target for chemoprevention. Nat Rev Cancer 7:139-47

23. Strizzi L, Catalano A, Vianale G et al (2001) Vascular endothelial growth factor is an autocrine growth factor in human malignant mesothelioma. J Pathol 193:468-75

24. Strizzi L, Vianale G, Catalano A et al (2001) Basic fibroblast growth factor in mesothelioma pleural effusions: correlation with patient survival and angiogenesis. Int J Oncol 18:1093-8

25. Harvey P, Clark IM, Jaurand MC et al (2000) Hepatocyte growth factor/scatter factor enhances the invasion of mesothelioma cell lines and the expression of matrix metalloproteinases. Br J Cancer $83: 1147-53$

26. Bielefeldt-Ohmann H, Marzo AL, Himbeck RP et al (1995) Interleukin-6 involvement in mesothelioma pathobiology: inhibition by interferon alpha immunotherapy. Cancer Immunol Immunother 40:241-50

27. Adachi Y, Aoki C, Yoshio-Hoshino N et al (2006) Interleukin-6 induces both cell growth and VEGF production in malignant mesotheliomas. Int J Cancer 119:1303-11

28. Fitzpatrick DR, Bielefeldt-Ohmann H, Himbeck RP et al (1994) Transforming growth factor-beta: antisense RNA-mediated inhibition affects anchorage-independent growth, tumorigenicity and tumor-infiltrating T-cells in malignant mesothelioma. Growth Factors 11:29-44

29. Dalbeth N, Lee YC (2005) Lymphocytes in pleural disease. Curr Opin Pulm Med 11:334-9

30. Fitzpatrick DR, Peroni DJ, Bielefeldt-Ohmann H (1995) The role of growth factors and cytokines in the tumorigenesis and immunobiology of malignant mesothelioma. Am J Respir Cell Mol Biol 12:455-60

31. Kim S, Buchlis G, Fridlender ZG et al (2008) Systemic blockade of transforming growth factor-beta signaling augments the efficacy of immunogene therapy. Cancer Res 68:10247-56

32. Hodgson JT, Darnton A (2000) The quantitative risks of mesothelioma and lung cancer in relation to asbestos exposure. Ann Occup Hyg 44:565-601

33. Kane AB (1998) Animal Models of Malignant Mesothelioma. In: Rom WN (ed) Environmental and occupational medicine. Lippincott-Raven, Boston, pp 377-386

34. Goodglick LA, Vaslet CA, Messier NJ et al (1997) Growth factor responses and protooncogene expression of murine mesothelial cell lines derived from asbestos-induced mesotheliomas. Toxicol Pathol 25:565-73

35. Smyth GK (2005) Limma: linear models for microarray data. In: Gentleman R (ed) Bioinformatics and computational biology solutions using $\mathrm{R}$ and Bioconductor. Springer Science+Business Media, New York, pp 397-420

36. Mohr S, Keith G, Galateau-Salle F et al (2004) Cell protection, resistance and invasiveness of two malignant mesotheliomas as assessed by $10 \mathrm{~K}$-microarray. Biochim Biophys Acta 1688:43-60

37. Okayasu R, Wu L, Hei TK (1999) Biological effects of naturally occurring and man-made fibres: in vitro cytotoxicity and mutagenesis in mammalian cells. Br J Cancer 79:1319-24

38. Hashimoto K, Morishige K, Sawada K et al (2005) Alendronate inhibits intraperitoneal dissemination in in vivo ovarian cancer model. Cancer Res 65:540-5

39. Cespedes MV, Espina C, Garcia-Cabezas MA et al (2007) Orthotopic microinjection of human colon cancer cells in nude mice induces tumor foci in all clinically relevant metastatic sites. Am J Pathol 170:1077-85

40. Tao K, Fang M, Alroy J et al (2008) Imagable $4 \mathrm{~T} 1$ model for the study of late stage breast cancer. BMC Cancer 8:228

41. Almand B, Clark JI, Nikitina E et al (2001) Increased production of immature myeloid cells in cancer patients: a mechanism of immunosuppression in cancer. J Immunol 166:678-89

42. Gabrilovich DI, Velders MP, Sotomayor EM et al (2001) Mechanism of immune dysfunction in cancer mediated by immature Gr-1+ myeloid cells. J Immunol 166:5398-406

43. Suzuki E, Kapoor V, Jassar AS et al (2005) Gemcitabine selectively eliminates splenic Gr-1+/CD11b+myeloid suppressor cells in tumor-bearing animals and enhances antitumor immune activity. Clin Cancer Res 11:6713-21

44. Ostrand-Rosenberg S, Sinha P (2009) Myeloid-derived suppressor cells: linking inflammation and cancer. J Immunol 182:4499-506

45. Sinha P, Clements VK, Bunt SK et al (2007) Cross-talk between myeloid-derived suppressor cells and macrophages subverts tumor immunity toward a type 2 response. J Immunol 179:977-83

46. Terabe M, Matsui S, Park JM et al (2003) Transforming growth factor-beta production and myeloid cells are an effector mechanism through which CD1d-restricted $\mathrm{T}$ cells block cytotoxic $\mathrm{T}$ lymphocyte-mediated tumor immunosurveillance: abrogation prevents tumor recurrence. J Exp Med 198:1741-52

47. Cheadle EJ, Riyad K, Subar D et al (2007) Eotaxin-2 and colorectal cancer: a potential target for immune therapy. Clin Cancer Res 13:5719-28

48. Qin XJ, Shi HZ, Huang ZX et al (2005) Interleukin-16 in tuberculous and malignant pleural effusions. Eur Respir J 25:605-11

49. Lenz G, Wright G, Dave SS et al (2008) Stromal gene signatures in large-B-cell lymphomas. N Engl J Med 359:2313-23

50. Ancuta P, Wang J, Gabuzda D (2006) CD16+ monocytes produce IL-6, CCL2, and matrix metalloproteinase-9 upon interaction with CX3CL1-expressing endothelial cells. J Leukoc Biol 80:1156-64

51. Hu Y, Ivashkiv LB (2006) Costimulation of chemokine receptor signaling by matrix metalloproteinase- 9 mediates enhanced migration of IFN-alpha dendritic cells. J Immunol 176:6022-33

52. Han IS, Ra JS, Kim MW et al (2003) Differentiation of CD34+ cells from human cord blood and murine bone marrow is suppressed by C6 beta-chemokines. Mol Cells 15:176-80 